\title{
Modular Total Absorption Spectrometer
}

\author{
M. Karny \\ Faculty of Physics, University of Warsaw, Warsaw PL-02-093, Poland \\ Joint Institute for Nuclear Physics and Application, Oak Ridge, TN 37831, USA \\ K.P. Rykaczewski \\ Physics Division, Oak Ridge National Laboratory, Oak Ridge, TN 37831, USA
}

A. Fijałkowska

Faculty of Physics, University of Warsaw, Warsaw PL-02-093, Poland

Joint Institute for Nuclear Physics and Application, Oak Ridge, TN 37831, USA

\section{B.C. Rasco}

Joint Institute for Nuclear Physics and Application, Oak Ridge, TN 37831, USA

Physics Division, Oak Ridge National Laboratory, Oak Ridge, TN 37831, USA

Dept. of Physics and Astronomy, Louisiana State University, Baton Rouge, LA 70803, USA

M. Wolińska-Cichocka

Physics Division, Oak Ridge National Laboratory, Oak Ridge, TN 37831, USA

Joint Institute for Nuclear Physics and Application, Oak Ridge, TN 37831, USA

Heavy Ion Laboratory, University of Warsaw, Warsaw PL-02-093, Poland

\section{R.K. Grzywacz}

Dept. of Physics and Astronomy, University of Tennessee, Knoxville, TN 37966, USA

Physics Division, Oak Ridge National Laboratory, Oak Ridge, TN 37831, USA

$$
\text { K.C. Goetz, D.Miller }{ }^{1}
$$

Dept. of Physics and Astronomy, University of Tennessee, Knoxville, TN 37966, USA

\section{E.F. Zganjar}

Dept. of Physics and Astronomy, Louisiana State University, Baton Rouge, LA 70803, USA

Abstract

Email address: karny@mimuw.edu.pl (M. Karny)

${ }^{1}$ Present address: Idaho National Laboratory, Idaho Falls, ID 83415, USA

Preprint submitted to Elsevier

August 16, 2016

(C) 2016. This manuscript version is made available under the Elsevier user license http://www.elsevier.com/open-access/userlicense/1.0/ 
The design and performance of the Modular Total Absorption Spectrometer built and commissioned at the Oak Ridge National Laboratory is presented. The active volume of the detector is approximately one ton of $\mathrm{NaI}(\mathrm{Tl})$, which results in very high full $\gamma$ energy peak efficiency of $71 \%$ at $6 \mathrm{MeV}$ and nearly flat efficiency of around $81.5 \%$ for low energy $\gamma$-rays between $300 \mathrm{keV}$ and $1 \mathrm{MeV}$. In addition to the high peak efficiency, the modular construction of the detector permits the use of a $\gamma$-coincidence technique in data analysis as well as $\beta$-delayed neutron observation.

\section{Introduction}

A need for total absorption spectrometry in the studies of complex $\beta$-decays has been recognised since the 1970's [1]. Since then, several versions of highefficiency total absorption spectrometers (TAS) have been built and utilised in $\beta-\gamma$ spectroscopy complementing the measurements using high energy resolution, but low efficiency Ge detectors. In particular, TAS measurements are mandatory for establishing the true $\beta$-decay pattern in fission products. One of the key phenomenon determining the decay properties of these neutron rich nuclei is the competition of allowed Gamow-Teller and first forbidden $\beta$ transitions. Large decay energy $\left(\mathrm{Q}_{\beta}\right)$ values result in many relatively weak $\beta$ transitions distributed over many final states in regions with high level energy and density. These $\beta$ transitions may be followed by many $\gamma$ transitions cascading to the ground or low energy isomeric state in the final nucleus. This is also known as Pandemonium effect [1]. To establish a true distribution of $\beta$ feeding through the measurement of $\beta$ and $\gamma$ radiation, very high detection efficiency is essential even at the expense of energy resolution. It was demonstrated in several cases that the $\beta$-feeding pattern derived from measurements using high-resolution, low-efficiency germanium detectors was incorrect, compare, e.g. $[2,3]$. In experiments using low efficiency $(<10 \%)$ Ge arrays, the $\beta$-feeding is obtained as a difference between intensity of apparent $\gamma$-rays feeding and de-exciting a given level in a daughter nucleus. Since many weak $\gamma$-transitions remain undetected, this results in erroneous reconstruction of $\beta$ feeding pattern. Also, in some cases there are weak, but high energy $\gamma$-transitions emitted after $\beta$-decay, which were beyond the detection range of low efficiency Ge setup. Typically, a decay scheme studied with relatively low efficiency detectors has an overestimated $\beta$-population of low energy levels and missing $\beta$-strength at high excitation energies $[2,3,4]$.

In the past 20 years experiments with different total absorption spectrometers have been performed at INEL [5], GSI-Darmstadt [6], ISOLDE facility [7] and Jyväskylä [8]. New TAS devices, like the Summing NaI(Tl) (SuN) detector [9] at the National Superconducting Cyclotron Laboratory and the DTAS detector designed for the future Facility for Antiproton and Ion Research (FAIR) [10], have been commissioned more recently and started data taking. 
Here, we present the newly constructed Modular Total Absorption Spectrometer (MTAS) built and commissioned at the Holifield Radioactive Ion Beam Facility at the Oak Ridge National Laboratory. MTAS was constructed to study the $\beta$-strength distribution in fission products relevant to reactor decay heat analysis [11] and properties of antineutrinos produced by the decay of fission products in nuclear reactors.

This article is structured as follows: section 2 describes the detector array, section 3 discusses GEANT4 simulations of the response functions and overall efficiency of MTAS, and a discussion of the new MTAS capabilities facilitated by its novel modular design is given in section 4 .

\section{MTAS Description}

\subsection{Geometry and control electronics}

The Modular Total Absorption Spectrometer, produced by Saint Gobain Crystals (Hiram, OH), consists of $18 \mathrm{NaI}(\mathrm{Tl})$ hexagonal modules. Each of the 18 modules is 21 " long and 6.93" wide (side-to-side). There is also one central module of the same length and cross section, but with a 2.5" hole drilled through, as shown in figure 1 . The total active $\mathrm{NaI}(\mathrm{Tl})$ mass is approximately one ton, making MTAS the largest and most efficient detector of this type currently in use. The crystals are arranged in a honeycomb like structure and the various modules are referred to as the Central (C) detector, the Inner ring (I) detectors, the Middle ring $(\mathrm{M})$ detectors and the Outer ring $(\mathrm{O})$ detectors. These assignments reflect the distance of each module to the central axis of the detector (see figure 1). Each $\mathrm{NaI}(\mathrm{Tl})$ crystal is enclosed in a carbon fiber housing $(0.81 \mathrm{~mm})$, with the stainless steel $(0.04 \mathrm{~mm})$, teflon $(0.5 \mathrm{~mm})$ and silicon putty $(2.025 \mathrm{~mm})$ layers. The through-hole of the Central detector has a similar housing with the exception of the silicon putty being only $0.68 \mathrm{~mm}$ thick. The Central detector is viewed by twelve 1.5" diameter ETI9102 Photo-Multipliers (PMT) produced by ET-Enterprises (Uxbridge, UK), six on each end, while the other 18 detectors are viewed by 5" diameter ETI9390 PMTs, one on each end of the crystal. It results in a total of 48 PMT signals. Signals are fed to the Pixie16, rev. D digital electronics developed by XIA LLC (Mountain View, Ca) [12] using 12 bit digitisation at 100 MSPS. Digital Data Acquisition system allows for the triggers mode of operation, very low signal threshold, and capability to work with high rates. The high voltage for the photomultiplier tubes is supplied by remotely controlled ISEQ EDS F130P 16 channels modules capable of supplying up to $3 \mathrm{kV}$ and $500 \mu \mathrm{A}$ per channel. The $\mathrm{HV}$ modules are housed in a Wiener mini-MPOD crate.

The performance of each individual crystal was verified with measurements using a ${ }^{137} \mathrm{Cs}$ source placed either at the centre of the crystal and 20 inches away from the module surface (broad beam) or just on the housing of the crystal in 20 different positions along the crystal, 1 inch apart. 


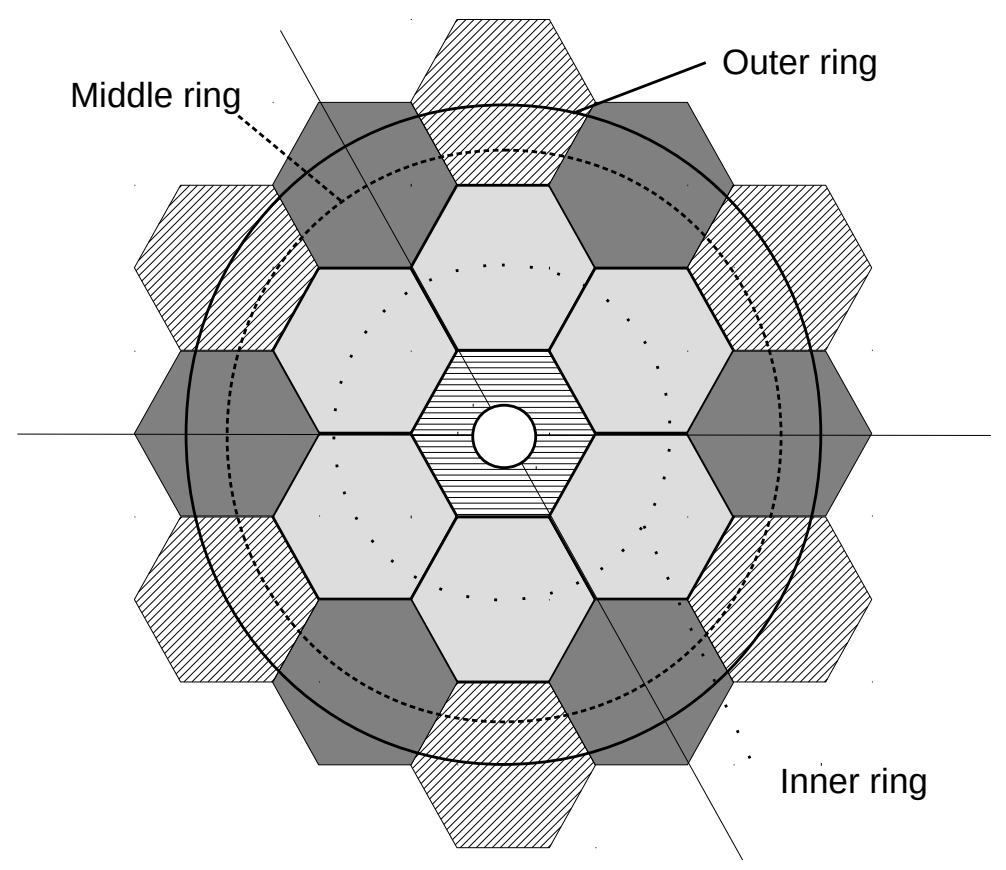

Figure 1: The honeycomb arrangements of the NaI modules in MTAS. The Inner-ring modules are filled with light grey. The Middle-ring modules with dark grey, while the Outer-ring modules are hatched a $45^{\circ}$, while the Central detector is hatched horizontally. 


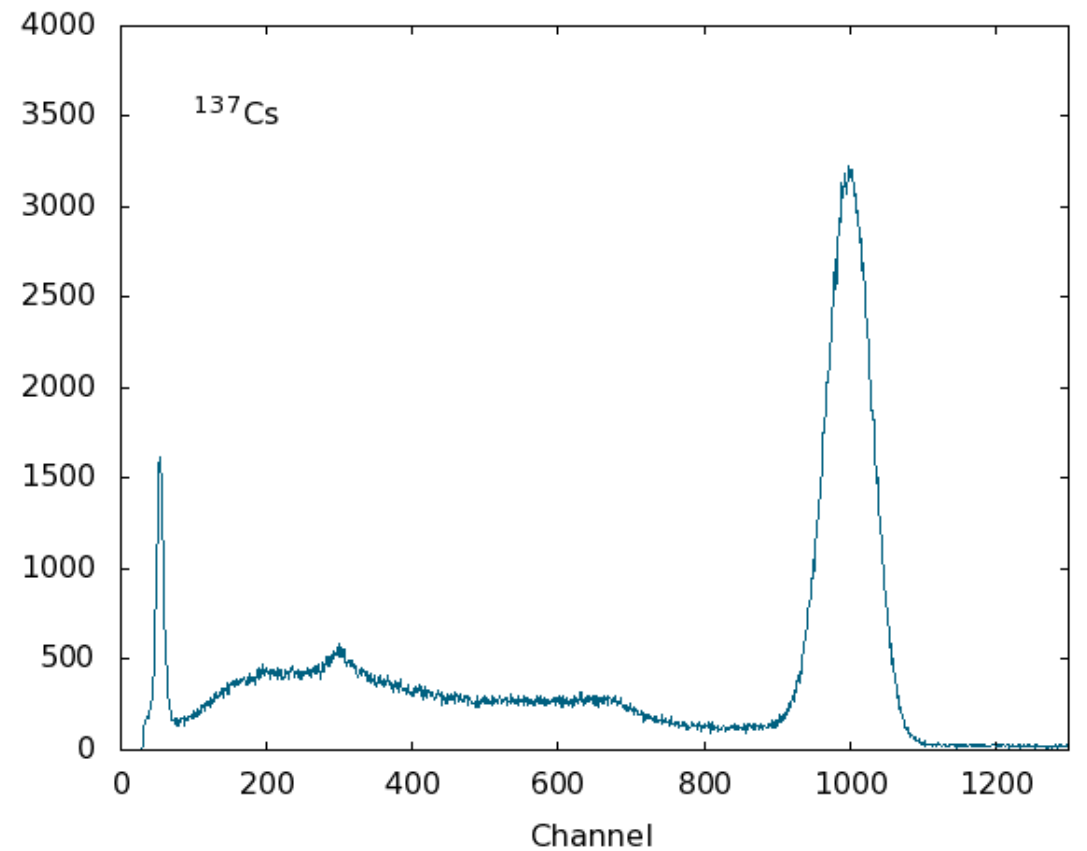

Figure 2: A typical ${ }^{137} \mathrm{Cs}$ source spectrum measured with a single MTAS module. The source was placed at the middle of the crystal and 20" away from the surface of the module (broad beam). Note the presence of $\approx 33 \mathrm{keV}$ barium X-rays to demonstrate the low energy threshold.

Figure 2 shows a typical response of one of the crystals to a broad beam $\gamma$-rays from ${ }^{137} \mathrm{Cs}$. Due to the minimal amount of material used to encapsulate the crystals the $\approx 33 \mathrm{keV} \mathrm{X}$-ray in daughter barium isotope can be detected. The full-width-at-half-maximum (FWHM) energy resolution at $662 \mathrm{keV}$ for all crystals excluding the Central one is between $6.4 \%$ and $7.9 \%$. Quoted values are for the summed spectra i.e.. photomultiplier signals from both ends of the crystals were added in software. Figure 3 shows the position (in channels) of $662 \mathrm{keV}$ line vs. ${ }^{137} \mathrm{Cs}$ source position along the side of the crystal. The position of the peak remains constant within $1 \%$ along the entire crystal while in the central part (starting 2-3" from the each side) uniformity is better then $0.2 \%$.

Because MTAS has such a large volume and high efficiency it is very important to shield it from background radiation. The count rate can easily reach 15000 events per second in the outside module, when not shielded and operating at the standard laboratory room background conditions. To reduce the rate due to natural background, MTAS has been surrounded by several layers of shielding. There are two layers of lead wool blankets made by Lancs Industries (Kirkland, Wa), each equivalent to $1 / 4$ " thick solid lead, directly on the support frame of MTAS. Further away from the detector, a lead cave has been constructed, consisting of 1" of lead on the top and sides. Standard 2" thick 


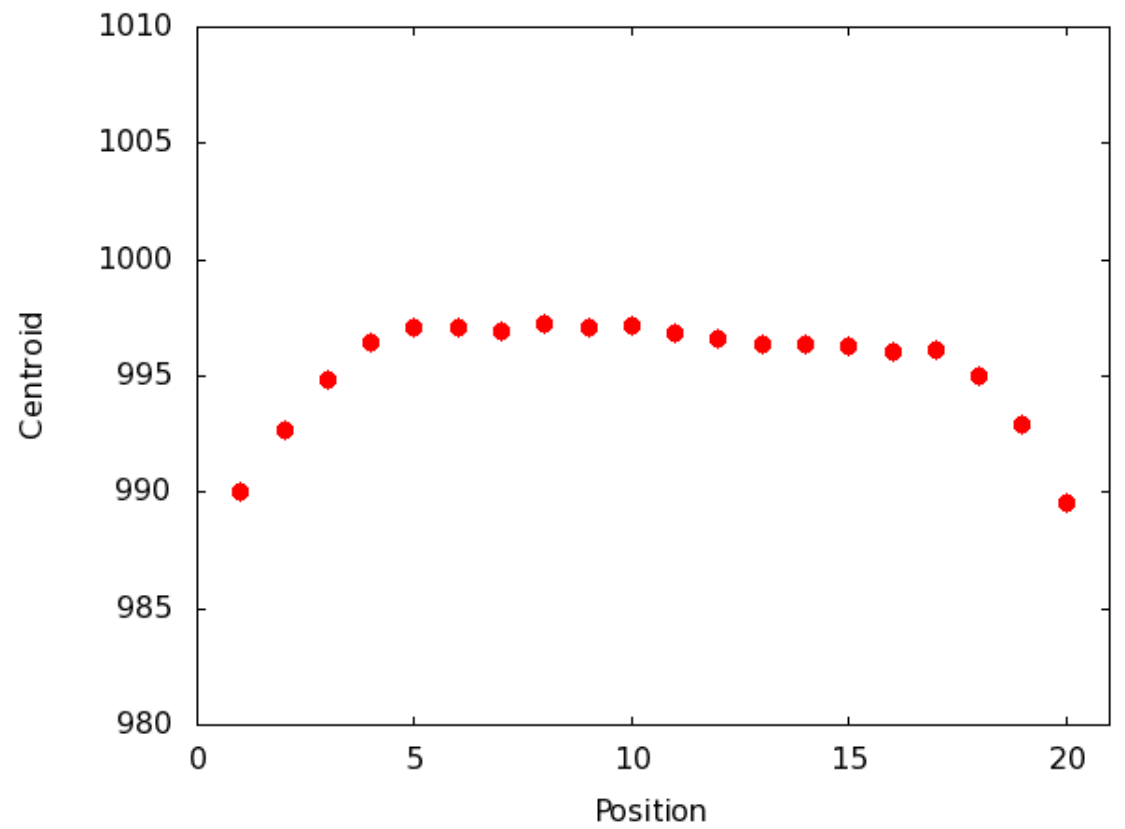

Figure 3: Amplitude of the $662 \mathrm{keV}$ peak (in channels) vs. the source position along the detector's main axis (in inches). The source was placed on one of the outside module's housing (i.e. not the Central detector). 
lead bricks are placed at the bottom of the detector, just beneath the crystals. The front and back side of the detector are covered with additional lead blankets adding up to an additional $\approx 1$ " solid lead equivalent. In addition MTAS has been fitted with neutron capturing rubber-like sheets ( $9 \%$ borated polyurethane, SWX-227A Flexi Panel produced by ShieldWerx) on the sides and at the top of the detector, space permitting. The resulting background count measured during the experiment, but with beam off the target, is 2400 counts per second for a summed spectrum in the energy range between $100 \mathrm{keV}$ and $10 \mathrm{MeV}$. This background, if needed, can be further suppressed by coincidence requirement with the silicon $\beta$-detectors described below.

\subsection{Auxiliary detectors}

In the current set-up, MTAS is equipped with two I-1000 $1 \mathrm{~mm}$ thick 7strip silicon detectors built by Micron Semiconductor LLC (Lancing, UK), for $\beta$-particle measurement. The active area of the detectors are is $60 \times 40 \mathrm{~mm}^{2}$ and are divided into 7 strips, $8.5 \mathrm{~mm}$ each, perpendicular to the central axis of MTAS. These two silicon detectors are placed close together and surround the radioactive source implanted on the moveable transport tape of the Moving Tape Collector (MTC) device designed and made at the Luisiana State University. The distance between silicon detectors is below $2 \mathrm{~mm}$. The solid angle coverage is $\approx 96 \%$ of $4 \pi$. During measurements performed by means of on-line isotope separation, nuclei of interest are implanted onto the tape in front of MTAS and then transported to the center of the detector using the MTC. Distance between the collection and measurement point is $\approx 36$ inches and can be traveled by the source in a minimum of $295 \mathrm{~ms}$. Standard operation mode is 500 to $780 \mathrm{~ms}$ for tape transport. The segmentation pattern of silicon detectors was chosen to enable position determination for each of the radioactive samples transported into the MTAS detector. Schematic drawing of the desired set-up presents figure 4 .

With the tape transport time around $700 \mathrm{~ms}$ all samples were stopped within a width of one $8.5 \mathrm{~mm}$ silicon strip. With the fastest transport time of $295 \mathrm{~ms}$, the accuracy of the source positioning was within three strips $(\approx 2 \mathrm{~cm})$ however stopping in the centre was still the most common outcome. Difference in the source positioning within $\approx 2 \mathrm{~cm}$ has no significant impact on the measured MTAS $\gamma$-spectrum and its de-convolution. The silicon detector FWHM of $32 \mathrm{keV}$ at $976 \mathrm{keV}$ electron energy was measured with an open ${ }^{207} \mathrm{Bi}$ electron source. The low energy threshold for electrons was estimated to be $\approx 70 \mathrm{keV}$.

\section{Geant simulation}

The knowledge of the response function of a complex detector like MTAS is required for the data analysis process [13]. The detector response can be measured using commercially available radioactive calibration sources, but this is not enough for accurate evaluation of the experimental data. Therefore, we use the GEANT4 [14] simulation package to calculate the response function based 


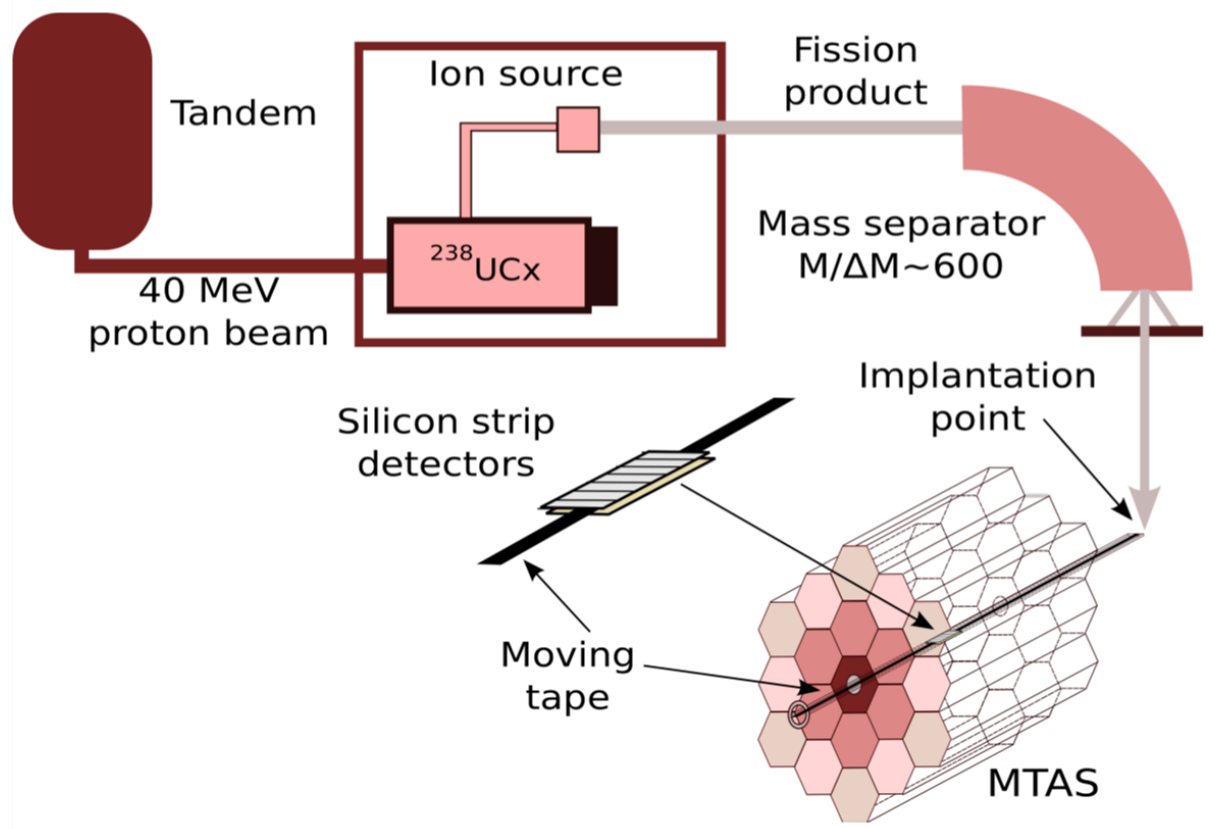

Figure 4: Schematic drawing of the MTAS setup as used for the HRIBF experiments. 


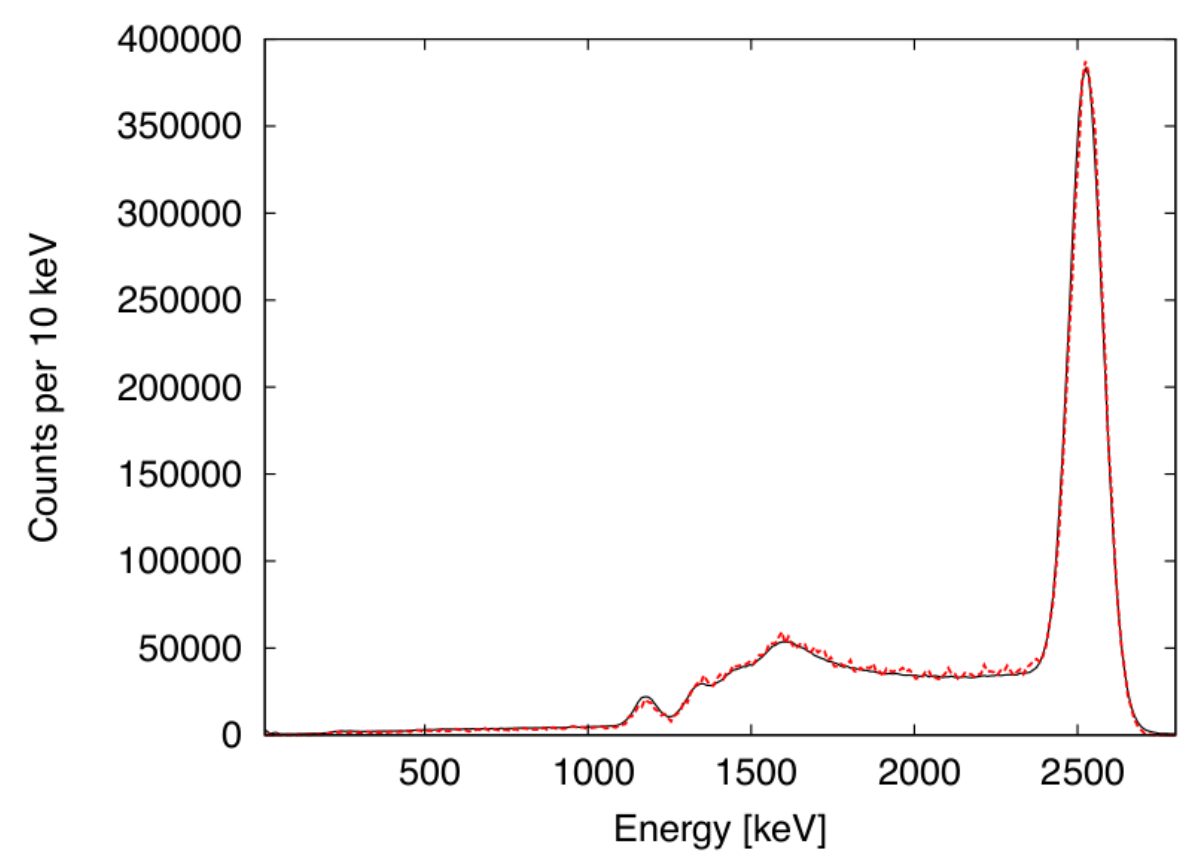

Figure 5: ${ }^{60}$ Co experimental spectrum as seen by MTAS (black) compared to the GEANT4 simulation (gray/ red(on-line)).

on known detector geometry. Simulations are verified by the comparison to the measured spectra of standard sources. Figures 5 and 6 present a comparison for the measurement with a ${ }^{60} \mathrm{Co}$ and ${ }^{90} Y$ sources for gamma and electron response functions, respectively. For simulations of the detector response function to $\gamma$ rays we used 4.9.4 and 4.10.1 versions of GEANT package with G4EMLOW6.19 and G4EMLOW6.41, respectively for low energy $\gamma$ cross section tables. For neutrons we use GEANT 4.10.1 with G4NDL4.5 tables. Two approaches to the simulation can be taken. First as described in [15] calculates the response function directly based on the light production in the scintillation crystal. Second approach described in [13] calculates the response function based on the energy deposited, light production and known FWHM of the detector. The latter approach allows for faster processing and has been adopted for the calculations described in this paper.

\subsection{Efficiency}

The main design goal of any total absorption spectrometer is to maximise its efficiency. There are three main variables in the process: the solid angle coverage, the amount of the material between the source and the active detector, and the volume of the detector. The solid angle coverage affects overall efficiency and is maximised by surrounding the source with the detector. The material 


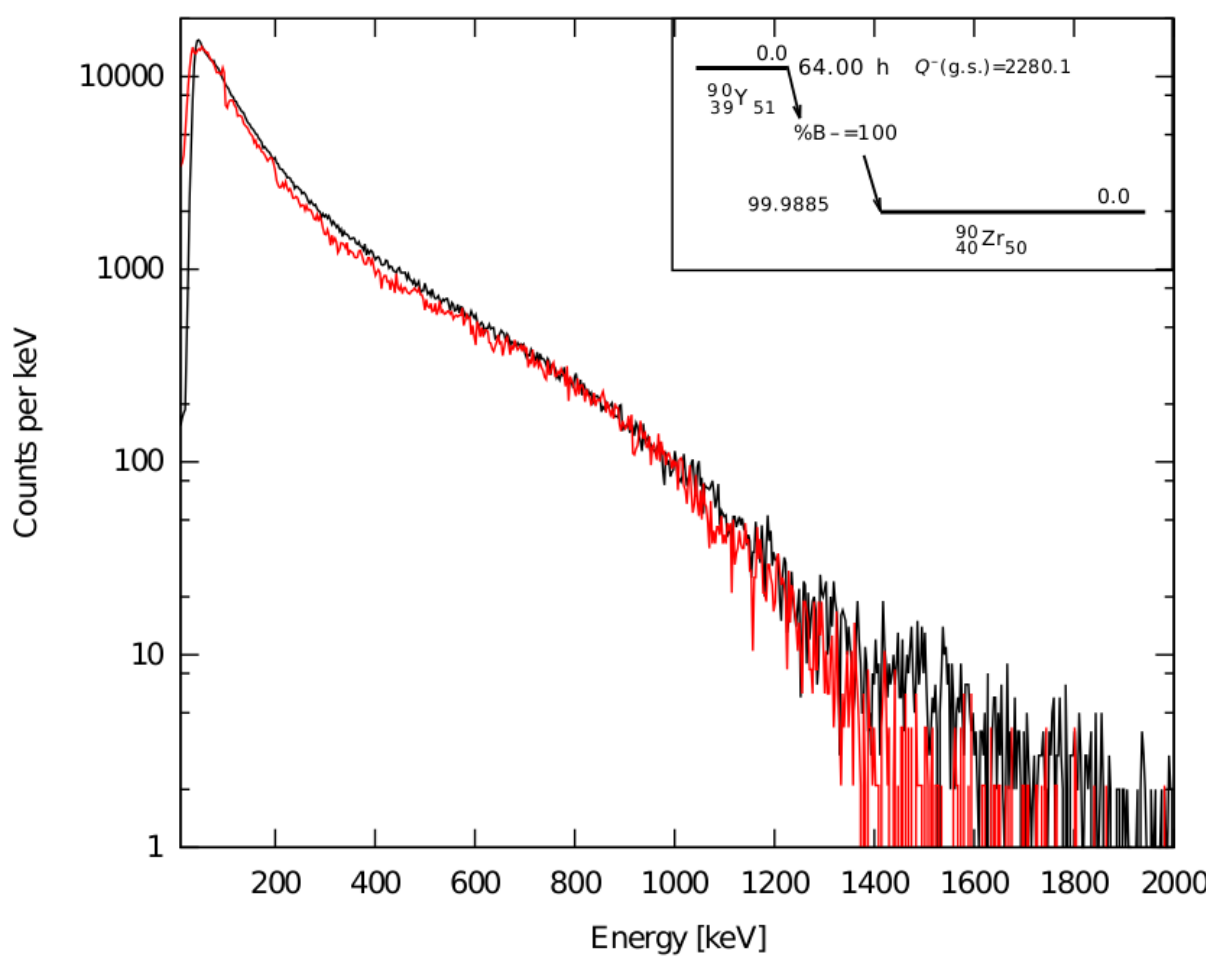

Figure $6:{ }^{90} \mathrm{Y} \beta$ decay experimental spectrum as seen by MTAS (black) compared to the GEANT4 simulation (gray/ red(on-line)). Clearly electrons from the decay have sufficient energy to enter NaI crystals, but can be simulated correctly. The inset shows the decay scheme. 
between the source and the active detector can be split into two components: housing material of the detector itself and any other material related to a given experimental set-up. Some examples of the materials include the auxiliary detectors, vacuum pipe walls and support structures for the tape system. While the $\mathrm{NaI}(\mathrm{Tl})$ crystal housing cannot be changed after initial optimisation and production, the surrounding matter can be optimised according to the particular needs of the experiment.

Figure 7 shows the calculated efficiency of bare MTAS (solid lines) and the MTAS detector fully equipped and ready for on-line measurement (dashed lines). Upper set of lines shows efficiency for all crystals, middle lines for Central crystal only, while bottom set of lines presents efficiency of the Inner ring detectors summed-up. Experimental points present measurements of ${ }^{137} \mathrm{Cs}$ and ${ }^{65} \mathrm{Zn}$ in bare MTAS detector configuration.

Full energy peak efficiency (i.e. the probability of registering the full energy of the incoming $\gamma$-ray) peaks at $81.5 \%$ for $500 \mathrm{keV}$ and goes down to $71 \%$ for $6 \mathrm{MeV}$ single $\gamma$-rays. A decrease of the maximum full energy peak efficiency for low energy $\gamma$-rays from $92.7 \%$ at $300 \mathrm{keV}$ to $81.5 \%$ at $500 \mathrm{keV}$ is noticeable, when calculations of bare and fully equipped MTAS are compared. Total efficiency (i.e. the probability of registering any portion of the incoming $\gamma$-ray energy not shown on the figure) of the MTAS detector with the $1 \mathrm{~mm}$ auxiliary silicon detectors reaches $98.9 \%$ at $500 \mathrm{keV}$.

Presented efficiencies are for mono energetic $\gamma$ transitions, to obtain the detection efficiency for the multiple $\gamma$ cascade respective mono energetic $\gamma$-ray efficiencies should be multiply. The higher multiplicity for the same summed energy, the lower summed peak efficiency gets. The total efficiency is much less sensitive to the multiplicity of $\gamma$ transition in the cascade.

The silicon detector efficiency for electrons, based on GEANT4 simulations, amounts to $94 \%$ when the $70 \mathrm{keV}$ threshold is taken into account for $1581 \mathrm{keV}$ mono-energetic electron energy. The geometric solid angle coverage is $96 \%$ (section 2.2 ).

\section{MTAS performance}

To date MTAS has been used to measure the $\beta$-decay of over $70{ }^{238} \mathrm{U}$ fission products, from the region between $\mathrm{Z}=34{ }^{85} \mathrm{Se}$ and $\mathrm{Z}=41{ }^{98} \mathrm{Nb}$, and from the region between $\mathrm{Z}=50{ }^{131} \mathrm{Sn}$ and $\mathrm{Z}=57^{146} \mathrm{La}$.

Detailed analysis and results will be published in forthcoming papers. In this article we present selected data to illustrate MTAS's performance and capabilities.

\subsection{Modularity}

The data analysis of a TAS like spectra requires a knowledge of the decay paths of the exited levels under consideration. This is usually done by incorporating the known partial level scheme into the analysis. If there is no enough

information, and in particular when new $\beta$ feedings at high excitation energies 


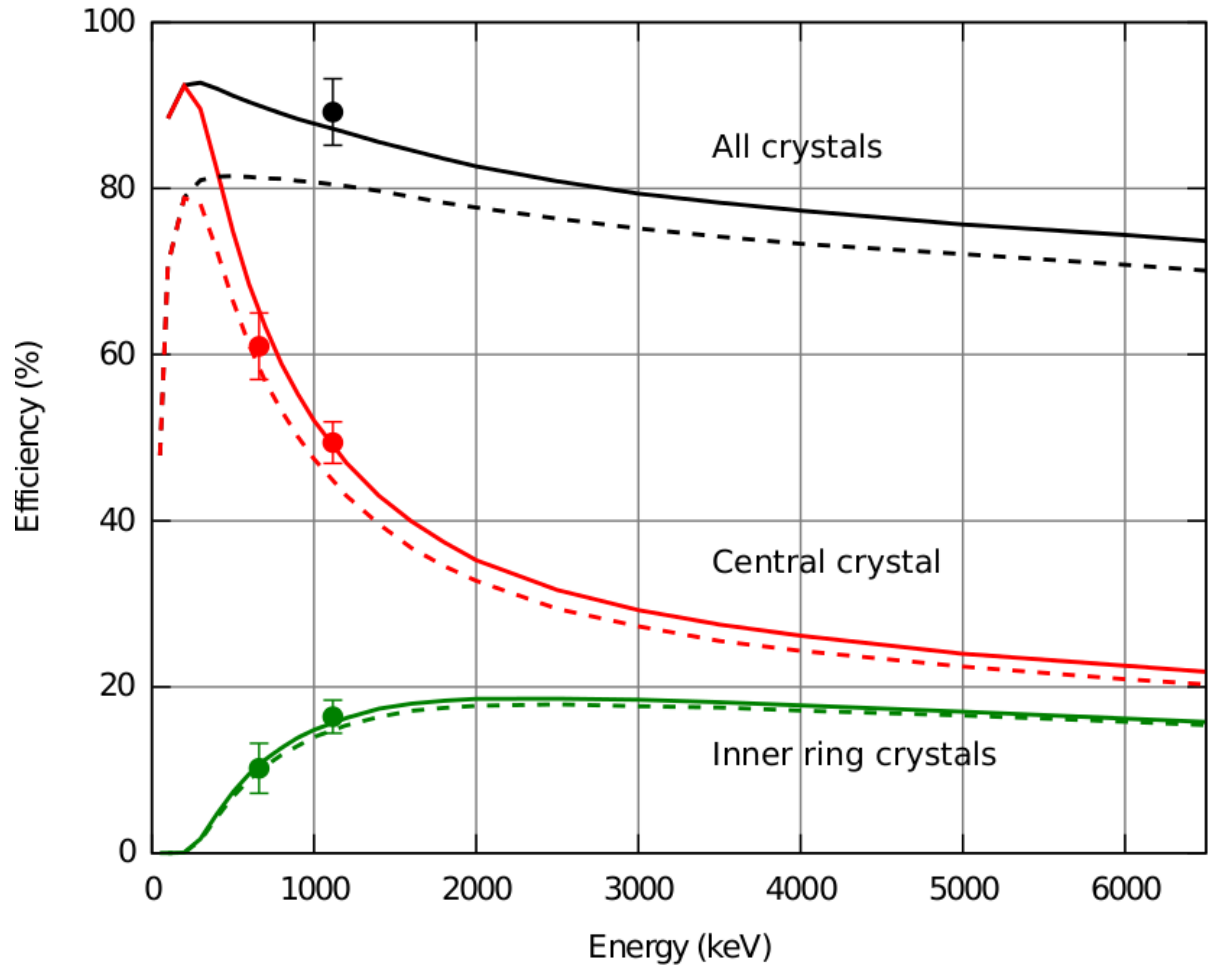

Figure 7: (Color online) Full energy absorption peak efficiency for MTAS with (dashed lines) and without any (solid lines) auxiliary detectors. The GEANT4 simulation: black (top) lines - all crystals, red (middle) lines - Central detector only, green (bottom) lines the Inner ring detectors only. Experimental data marked with points were measured for the setup without any auxiliary detectors. 
is observed, new levels represented by respective energy bins are added. The decay paths of those bins is approximated by the assumptions, that they decay by E1, E2 or M1 transitions to the know levels. The spin of the bin is calculated based on the Gamow-Teller selection rules and statistical model level density $[16,17,18]$. This is a procedure used in data analysis for a single crystal TAS spectrometers. However, in the case of Modular Total Absorption Spectrometer its modularity allows for the inclusion of coincidence spectra in the data analysis as a source of information on the decay paths of new level/bin. Figure 8 shows the two dimensional spectrum of the decay of ${ }^{86} \mathrm{Br}$ with the total energy plotted on the $\mathrm{Y}$-axis and the energy registered in the Central detector on the $\mathrm{X}$-axis (see also [19]). The maxima along the $\mathrm{X}$-axis for the given $\mathrm{Y}$-axis energy correspond to the strongest $\gamma$-rays de-exciting that bin or level. For example, at $\approx 4.3 \mathrm{MeV}$ on the Y-axis, there are a series of maxima at $\approx 0.5 \mathrm{MeV}, 1.2 \mathrm{MeV}$, 1.4 MeV, $2 \mathrm{MeV}, 2.3 \mathrm{MeV}, 2.7 \mathrm{MeV}$ and $2.9 \mathrm{MeV}$ on the X-axis. This corresponds to a very strongly $\beta$ fed level (51\%, according to the ENSDF data base) at $4.315 \mathrm{MeV}$ in ${ }^{86} \mathrm{Br}$ along with the associated $\gamma$-rays in the cascade de-exciting that level. Further discussion of the ${ }^{86} \mathrm{Br}$ decay will be presented in a dedicated paper.

Another important advantage of a modular device is the opportunity to analyse the spectra taken by a single module at each ring and/or rings of modules to verify the deconvolution of the total MTAS spectrum. Figure 9 shows the experimental $\beta$-decay spectrum of ${ }^{86} \mathrm{Br}$ as registered individually by the single crystals of MTAS excluding Central detector ( $\beta$-gated singles spectrum) in comparison to the relevant simulated spectrum. Central detector data are excluded from the spectrum as this crystal acts a small TAS-like device registering a sum of energies deposited by many $\gamma$-rays in the cascade. Unlike the total absorption MTAS spectrum of figure 10, the peaks in the spectrum displayed in figure 9 correspond to the single $\gamma$ transitions. The presented simulations (in figure 9) were based on data available from the ENSDF database as well as the results of the data analysis of the total MTAS spectrum (see figure 10). Therefore, spectra on figures 9 and 10 are not fitted independently. First, fit to the total spectrum is made and the corresponding single gamma spectrum is generated and compared to the experimental data. If large discrepancies are spotted on figure 9 in second step $\gamma$-ray intensities are changed and the simulation of both total and single like spectra is performed. This is an iterative procedure performed until acceptable agreement for both spectra is achieved.

The modularity of MTAS also impacts the construction of its response function and thus influencees the method of data analysis. In case of mono-crystal devices, the standard procedure involves simulations of the response function of the detector to the mono energetic $\gamma$-rays in the range of a few tens of $\mathrm{keV}$ to a few $\mathrm{MeV}$ with the lowest possible step size (i.e. $20 \mathrm{keV}$ ). From there the response for each level is constructed. The response function $\left(\mathbf{r}_{j}\right)$ of the $j$-th level is calculated as a convolution of the response function of a mono energetic $\gamma$-ray $\left(\mathbf{g}_{j k}\right)$ transition de-exciting level $j$ to level $k$ and an already calculated response function of the $k$-th level $\left(\mathbf{r}_{k}\right)$. Finally, the response is summed over 


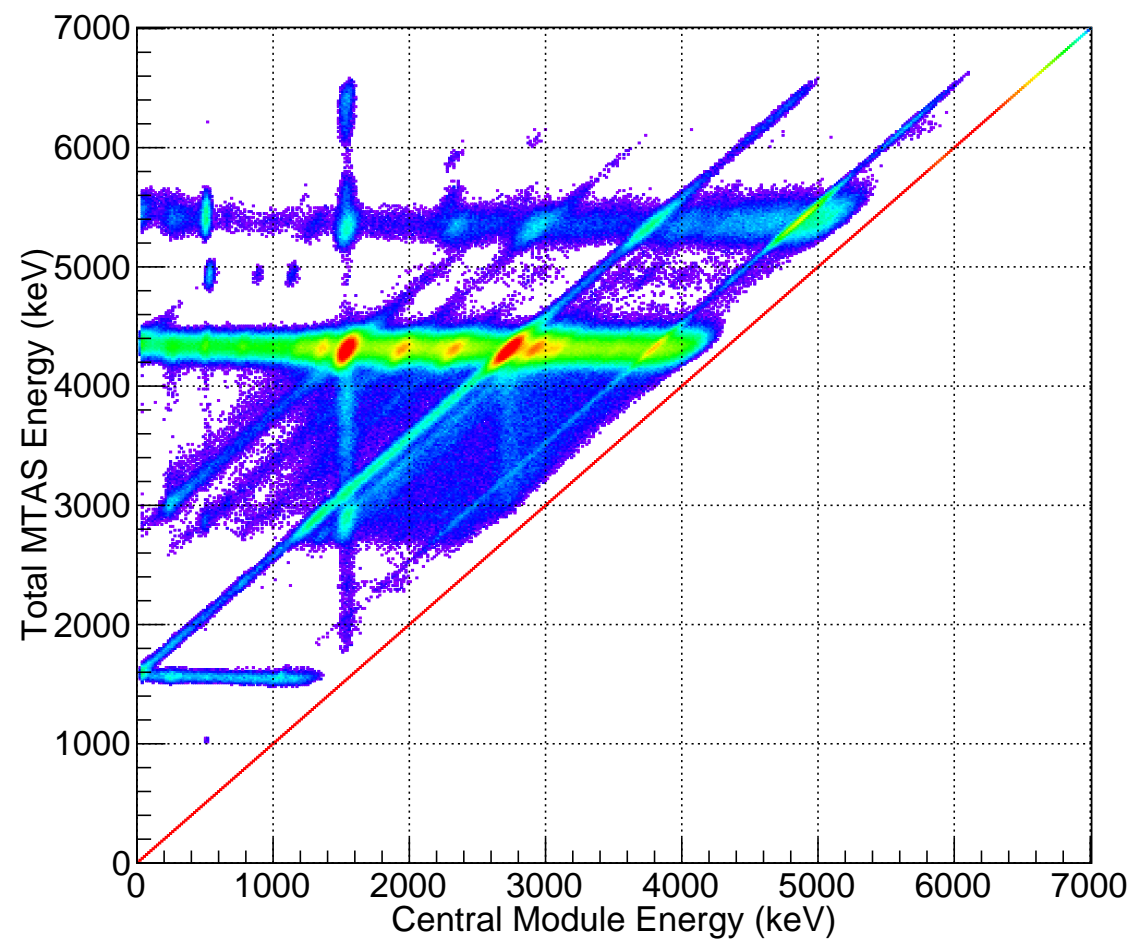

Figure 8: Two dimensional spectra of ${ }^{86} \mathrm{Br}$ decay. The $\gamma$-ray energies from Central module are plotted on the $\mathrm{X}$-axis, while sum of all MTAS detectors is plotted on the Y-axis. 


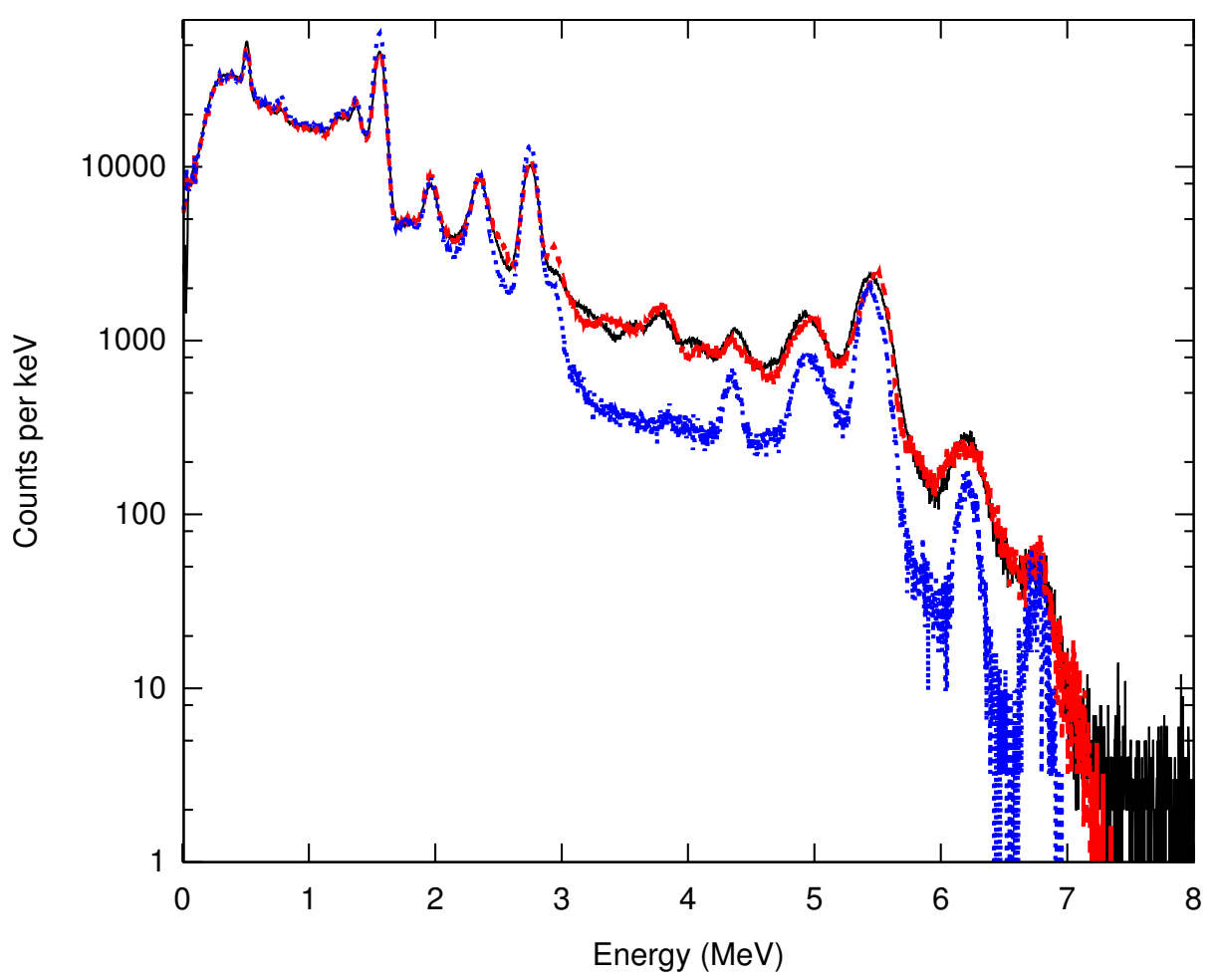

Figure 9: Experimental $\beta$-delayed $\gamma$ spectrum from the decay of ${ }^{86} \mathrm{Br}$ as registered by all single crystals of MTAS detector excluding Central (solid black) in comparison to the simulated spectrum from ENSDF database (dotted blue) and the result of the complete MTAS data evaluation (dashed red). 


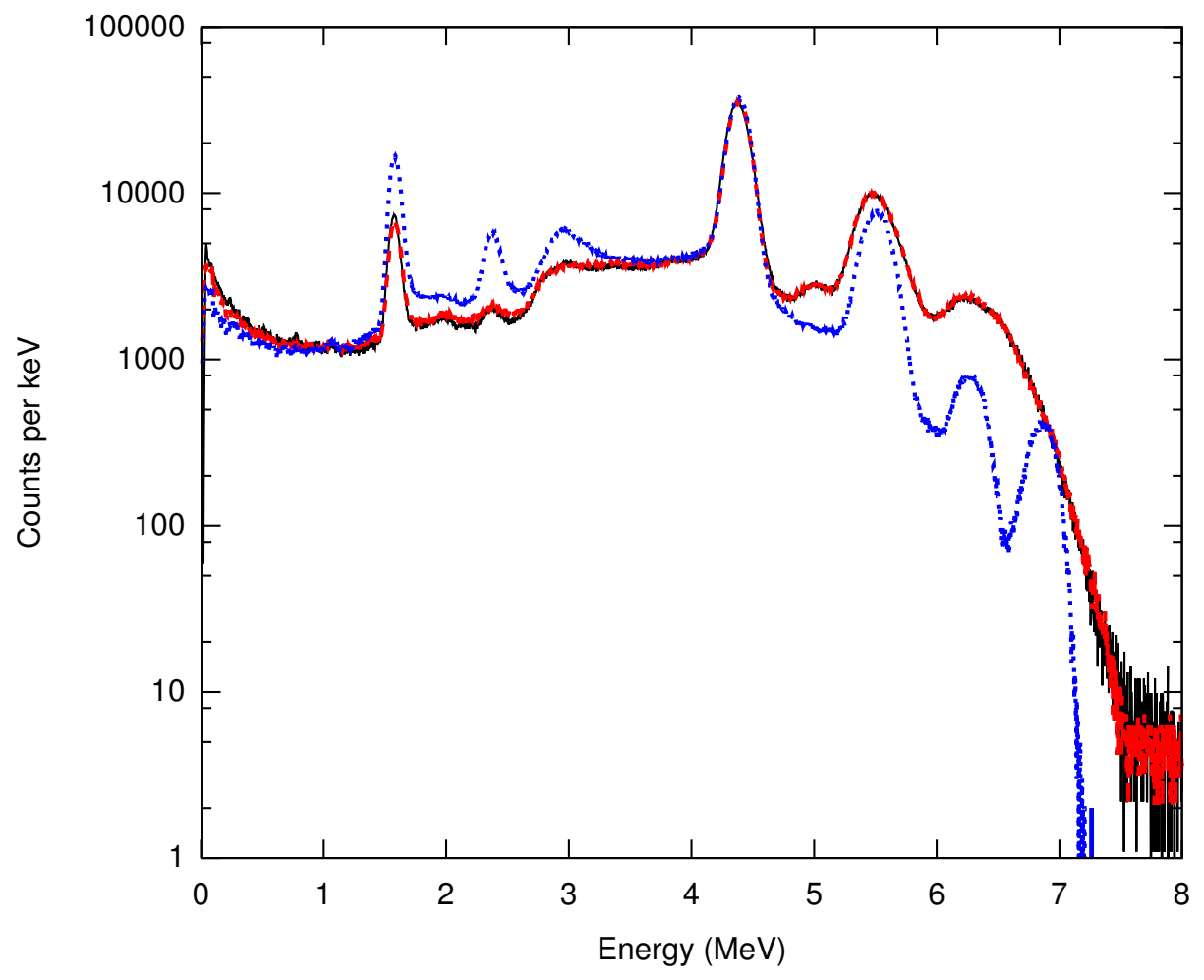

Figure 10: Experimental total MTAS $\beta$ delayed $\gamma$ spectrum from the decay of ${ }^{86} \mathrm{Br}$ (solid black) in comparison to the simulated spectrum from ENSDF database (dotted blue) and the result of the complete MTAS data analysis (dashed red). 
all possible $\gamma$-transitions de-exciting the level $j$ [13].

$$
\mathbf{r}_{j}=\sum_{k=0}^{j-1} b_{j k} \mathbf{g}_{j k} \otimes \mathbf{r}_{k}
$$

where $b_{j k}$ is a branching ratio for the transition between levels $j$ and $k$. In addition, the resulting $\mathbf{r}_{j}$ has to be convolved with the response function of the detector to $\beta^{-}$electrons $\left(\mathbf{b}^{-}\right)$emitted during $\beta$ decay to the fed level $j . \mathbf{R}_{j}^{\beta-}=$ $\mathbf{b}^{-} \otimes \mathbf{r}_{j}$. This method requires extensive initial computing effort to prepare the $\gamma$ response functions $\mathbf{g}$ of the detector but later benefits from the fast operation of convolution. In the case of a modular spectrometer, in spite of initial gain matching all MTAS segments may exhibit gain drifts in time which will result in slightly different calibration and parametrisation of the FWHM needed to match the experimental spectrum. Therefore in our data analysis we construct the level response function directly through simulation GEANT4. The same calibrations, coincidence and threshold requirements used during the experiments are used to sort simulated events into spectra. With modern computers this approach is fast enough to be acceptable. It is also very convenient when changes to the auxiliary detectors are made between experiments, as data analysis can be started immediately without the delay needed to build the required $\gamma$ response data base.

\subsection{MTAS sensitivity to the $\beta$ transitions with low beta end-point}

One of the main goals of $\beta$ decay spectroscopy is to measure $\beta$ transitions to all levels including highly excited ones, where the energy window for $\beta$ decay is small. This is especially important for neutron rich isotopes where allowed GT transitions connect the ground state of the parent to highly excited levels in the daughter, with only a tail of the GT-resonance within $\beta$ decay energy window.

The MTAS array measures $\beta$-gated and un-gated spectra. The latter are usually heavily contaminated by laboratory background radiation and is not used in the data analysis. Despite the gain from a spectra practically free from laboratory background, the $\beta$-gated MTAS spectra does show a decrease in sensitivity at the excitation energies close to the $Q_{\beta}$ value.

Figure 11 shows the high energy part of an MTAS $\beta$-gated and un-gatedbackground subtracted spectrum from ${ }^{110 m} \mathrm{Ag}$ decay. The $\beta$-decay feeding to the level just $90 \mathrm{keV}$ below the $Q_{\beta}$ value is visible in the un-gated spectrum, while appearing very weak in the $\beta$-gated spectrum. Although $90 \mathrm{keV}$ is above the established $70 \mathrm{keV}$ silicon detector threshold one has to remember that $90 \mathrm{keV}$ is an end-point of the continuous $\beta$ spectra with most of the electrons energy being below $70 \mathrm{keV}$, and thus the majority of the electrons in this decay are below the threshold of the Si-detectors. Although the effect is taken into account by the simulation one has to remember that due to this effect established feedings to the levels close to the $Q_{\beta}$ will have larger systematic uncertainties then others. In both spectra pile-up events are visible above the $Q_{\beta}$ value of $3.01 \mathrm{MeV}$. Events below $700 \mathrm{keV}$ peak in un-gated but background subtracted spectrum (dottedred) are residues after subtraction of the large background contribution in this 


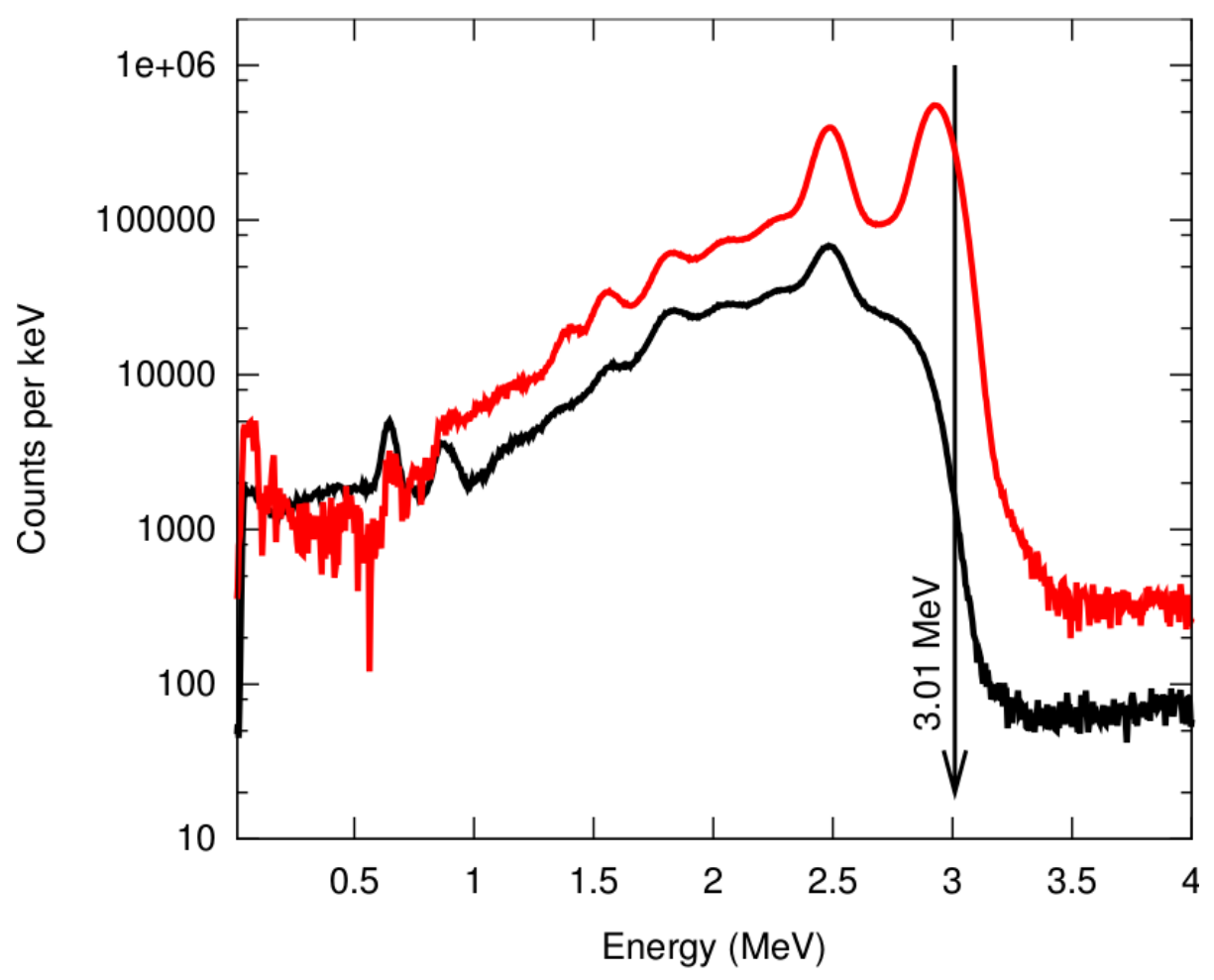

Figure 11: The experimental $\beta$ decay spectrum of ${ }^{110 m} \mathrm{Ag}\left(Q_{\beta}=3.01 \mathrm{MeV}\right) . \quad \beta$-gated black, ungated-background subtracted - dashed red. The excess of counts around channel $3000(\approx 3 \mathrm{MeV})$ illustrates the impact of silicon detection thresholds for $\beta$ particles on MTAS spectrum.

area comparable to the resulting uncertainties. Slight difference between spectra (i.e. around $1 \mathrm{MeV}, 1.5 \mathrm{MeV}$ ) come mainly from the fact that some $\gamma$-rays undergo Compton scattering inside relatively thick silicon detectors producing a false "beta" signal. Conversion electrons produced in the decay of the ${ }^{110 m} \mathrm{Ag}$ also can produce the "false" beta signal for gating. Both processes enhance probability of registering some decay paths and thus changing the shape of the $\beta$-gated spectrum when compared to the un-gated spectrum.

\section{3. $\beta$-delayed neutrons}

Fission products often have large $\beta$-decay energies exceeding the neutron separation energy in the daughter nucleus. This results in $\beta$-delayed neutron emission $(\beta \mathrm{n})$ releasing a part of the available decay energy. For analysis of total absorption spectroscopy data, information about $\beta \mathrm{n}$ branching is required in order to properly normalise the $\beta \gamma$ part of the decay. The emitted neutron undergoes elastic and in-elastic s-wave scattering and $\left(n, n^{\prime} \gamma\right)$ scattering in the 
$\mathrm{NaI}$ which slows it down. Due to the large volume of MTAS, this process is fast enough to slow down neutrons to the energies sufficiently low to allow for an efficient capture of the neutron, mostly on the iodine isotope component of $\mathrm{NaI}(\mathrm{Tl})$. This produces the reaction ${ }^{127} \mathrm{I}(n, \gamma){ }^{128} \mathrm{I}^{*}$. A neutron is captured at levels above the neutron separation energy of ${ }^{128} \mathrm{I} S_{n}=6826.13(5) \mathrm{keV}$ mainly at the $6826 \mathrm{keV}$ level. Levels are then de-excited by many $\gamma$-rays. These $\gamma$ rays are registered in MTAS, producing peaks at and above 6.8 MeV. Figure 12 shows the MTAS spectrum of ${ }^{137}$ I decay with $\gamma \mathrm{s}$ from $(n, \gamma)$ capture visible around and above $6.8 \mathrm{MeV}$ (6.8 MeV from capture plus some neutron kinetic energy registered by MTAS from neutron scattering process). With GEANT4 simulations we compute the response function of the MTAS detector to mono energetic neutrons of different kinetic energies and fit it to the MTAS spectrum. The resulting $\beta$-delayed neutron intensity spectrum per $100 \mathrm{keV}$ vs. neutron energy is presented in the inset of figure 12 . The shape of the neutron intensity spectrum agrees well with the detailed experimental studies [20,21].

More information related to $\beta$-delayed neutron component which can be ex-

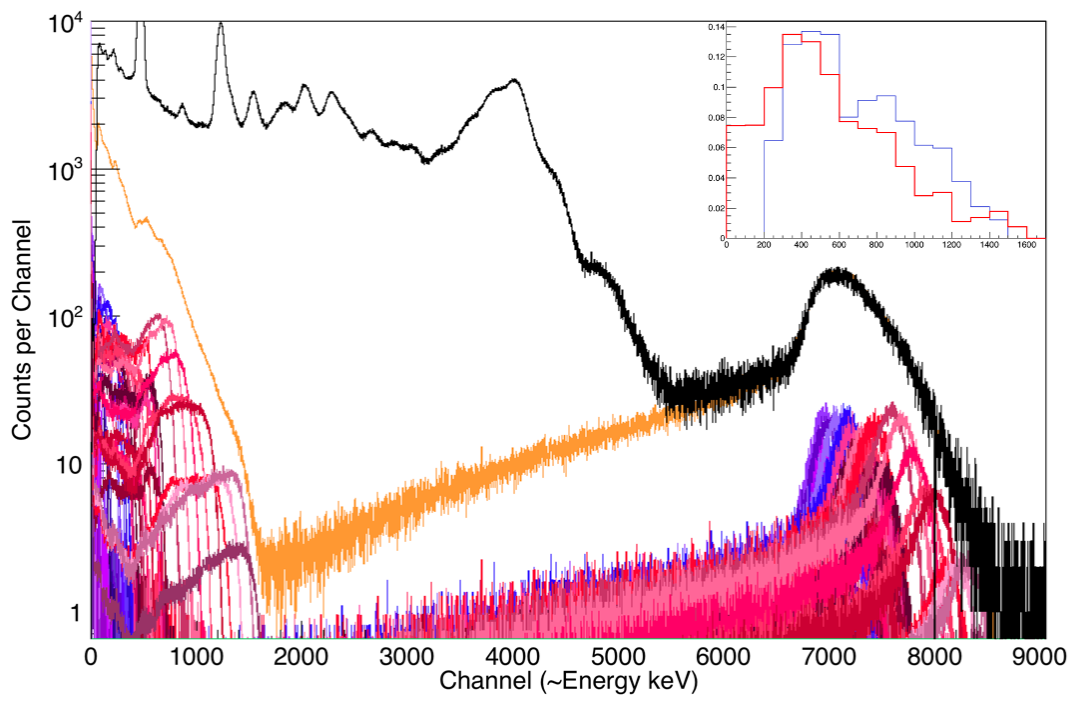

Figure 12: The MTAS spectrum of ${ }^{137}$ I decay (black) with the simulated response function of MTAS to the $\beta$ - delayed neutrons (yellow - all $\beta n$ neutrons, other colours - mono energetic neutrons). Inset shows the resulting neutron intensity per $100 \mathrm{keV}$ vs. neutron energy (red) in comparison to the literature data based on [20] (blue).

tracted from the MTAS data analysis are $\beta n$ branching ratios to the excited states in the $\beta$-n daughter nucleus. Figure 13 shows three spectra: black-solid MTAS total spectrum, purple -the Central detector spectrum for ${ }^{89} \mathrm{Br} \beta$ decay obtained under the following conditions: a) coincidence with silicon detector 
signal (presumably electron registration from $\beta$ decay), and $6.5 \mathrm{MeV}$ to $Q_{\beta}$ energy gate on the Inner, Middle and Outer ring detectors (presumably events related to $(\mathrm{n}, \gamma))$. The latter condition presents the yellow spectrum. The two peaks in the dashed-yellow spectrum at $\approx 0.8 \mathrm{MeV}$ and the other barely visible peak at $\approx 1.6 \mathrm{MeV}$ correspond to the excitation energy of first two excited states $(775 \mathrm{keV}, 1577 \mathrm{keV})$ in ${ }^{88} \mathrm{Kr}$. The low energy peaks in the range of $50-400 \mathrm{keV}$ come from neutron scattering in the Central detector. Although MTAS detector is not designed for precision neutron spectroscopy, the capability of registering and analysing the $\beta$-delayed neutron contribution of the decay increases the confidence in the final results of $\beta$-strength analysis.

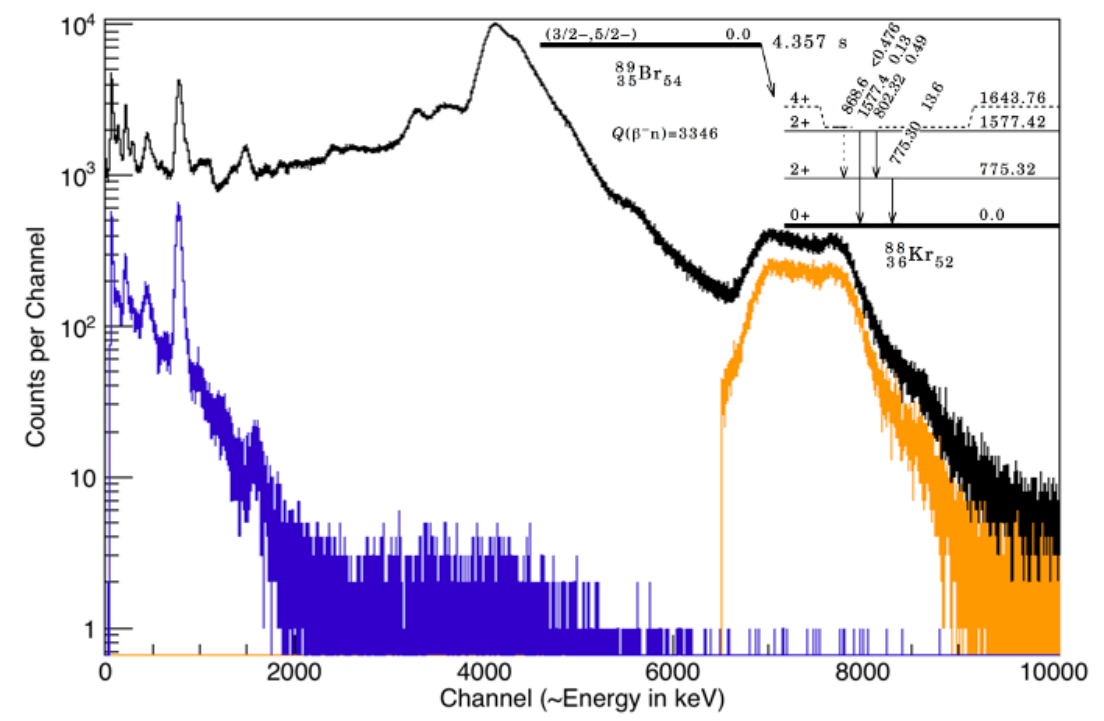

Figure 13: $\beta$-delayed $\mathrm{n}-\gamma$ transitions from the decay of ${ }^{89} \mathrm{Br}$ as measured with MTAS. The total MTAS spectrum is shown in solid-black, the total MTAS spectrum, if there is a large energy deposit (greater than $6.5 \mathrm{MeV}$ of energy) in the I, M, and O-ring events is shown in yellow, and the Central detector energy spectrum, given a large I, M, O-ring energy deposit, is shown in purple. A large energy deposit in the I, M, and O rings is indicative of a neutron capture in MTAS. The peaks first at $\approx 750 \mathrm{keV}$ and second barely visible at $\approx 1600 \mathrm{keV}$ correspond to the excitation energy of first two excited states $(775 \mathrm{keV}, 1577 \mathrm{keV})$ in ${ }^{88} \mathrm{Kr}$. The other low energy peaks in the range of $50-400 \mathrm{keV}$ come from $\left(n, n^{\prime} \gamma\right)$ scattering in Central detector. The inset shows the simplified decay scheme.

\section{Summary}

We have presented the Modular Total Absorption Spectrometer designed and commissioned at the Oak Ridge National Laboratory for $\beta$-decay studies of neu- 
tron rich nuclei relevant to decay heat and reactor neutrino anomaly problems. MTAS is currently the world-largest active volume and most segmented detector amongst total absorption spectrometers. MTAS's high efficiency, reaching $71 \%$ peak efficiency for $6 \mathrm{MeV} \gamma$-ray, is critical for measurements of $\beta$-decay to the highly excited states populated in Gamow-Teller $\beta$-transitions in fission products. The current set-up is designed for measurements at the on-line mass separators. However, the modular construction around its central opening and very high $\gamma$-detection efficiency make it an attractive detector for discovery studies of very weakly produced new nuclei at fragmentation facilities. This will be best achieved after an implementation and/or $\beta$ decay auxiliary detectors are fitted.

This work was supported by the US DOE, Office of Nuclear Physics through the contract No. DE-AC05-00OR22725, the US DOE award no. DE-FG0296ER40978, and by U.S. Department of Energy NNSA under the Stewardship Science Academic Alliance program through DOE Cooperative Agreement No. DE-FG52-08NA28552, and by the Office of Science, U.S.DOE through contracts no. DE-FG02-96ER40983 and DOE DE-SC0007431, and by the Polish National Centre for Science under the contract No. UMO-2013/08/T/ST2/00624.

\section{References}

[1] J. C. Hardy, L. C. Carraz, B. Jonson, P. G. Hansen, The essential decay of pandemonium: A demonstration of errors in complex beta-decay schemes, Phys. Lett. B 71 (1977) 307.

[2] Z. Hu, L. Batist, J. Agramunt, A. Algora, B. A. Brown, D. Cano-Ott, R. Collatz, A. Gadea, M. Gierlik, M. Górska, H. Grawe, M. Hellström, Z. Janas, M. Karny, R. Kirchner, F. Moroz, A. Płochocki, M. Rejmund, E. Roeckl, B. Rubio, M. Shibata, J. Szerypo, J. L. Tain, V. Wittmann, $\beta$ decay of ${ }^{97} \mathrm{Ag}$ : Evidence for the Gamow-Teller resonance near ${ }^{100} \mathrm{Sn}$, Phys. Rev. C 60 (1999) 024315.

[3] A. Algora, B. Rubio, D. Cano-Ott, J. L. Taín, A. Gadea, J. Agramunt, M. Gierlik, M. Karny, Z. Janas, A. Płochocki, K. Rykaczewski, J. Szerypo, R. Collatz, J. Gerl, M. Górska, H. Grawe, M. Hellström, Z. Hu, R. Kirchner, M. Rejmund, E. Roeckl, M. Shibata, L. Batist, J. Blomqvist, Fine structure of the Gamow-Teller resonance revealed in the decay of ${ }^{150} \mathrm{Ho} 2^{-}$isomer, Phys. Rev. C 68 (2003) 034301.

[4] M. Karny, L. Batist, B. Brown, D. Cano-Ott, R. Collatz, A. Gadea, R. Grzywacz, A. Guglielmetti, M. Hellström, Z. Hu, Z. Janas, R. Kirchner, F. Moroz, A. Piechaczek, A. Płochocki, E. Roeckl, B. Rubio, K. Rykaczewski, M. Shibata, J. Szerypo, J. Tain, V. Wittmann, A. Wöhr, Beta decay of ${ }^{103} \mathrm{In}$ : Evidence for the Gamow-Teller resonance near ${ }^{100} \mathrm{Sn}$, Nuclear Physics A 640 (1) (1998) 3-23. 
[5] R.C. Greenwood, D.A. Struttmann, Use of a Total Absorption Gamma-Ray Spectrometer to Measure Ground-State $\beta$-Branching Intensities, Nuclear Instruments and Methods A 317 (1992) 175.

[6] M. Karny, J. Nitschke, L. Archambault, K. Burkard, D. Cano-Ott, M. Hellström, W. Hüller, R. Kirchner, S. Lewandowski, E. Roeckl, A. Sulik, Coupling a total absorption spectrometer to the GSI on-line mass separator, Nuclear Instruments and Methods B 126 (1997) 411-415.

[7] E. Poirier, F. Maréchal, P. Dessagne, A. Algora, M. J. G. Borge, D. CanoOtt, J. C. Caspar, S. Courtin, J. Devin, L. M. Fraile, W. Gelletly, G. Heitz, A. Jungclaus, G. Le Scornet, C. Miehé, E. Nácher, B. Rubio, P. Sarriguren, J. L. Tain, O. Tengblad, C. Weber, (the ISOLDE Collaboration), B(GT) strength from $\beta$-decay measurements and inferred shape mixing in ${ }^{74} \mathrm{Kr}$, Physical Review C (69) (2004) 034307.

[8] A. Algora, D. Jordan, J. L. Tain, B. Rubio, J. Agramunt, A. B. PerezCerdan, F. Molina, L. Caballero, E. Nácher, A. Krasznahorkay, M. D. Hunyadi, J. Gulyás, A. Vitéz, M. Csatlós, L. Csige, J. Äysto, H. Penttilä, I. D. Moore, T. Eronen, A. Jokinen, A. Nieminen, J. Hakala, P. Karvonen, A. Kankainen, A. Saastamoinen, J. Rissanen, T. Kessler, C. Weber, J. Ronkainen, S. Rahaman, V. Elomaa, S. Rinta-Antila, U. Hager, T. Sonoda, K. Burkard, W. Hüller, L. Batist, W. Gelletly, A. L. Nichols, T. Yoshida, A. A. Sonzogni, K. Peräjärvi, Reactor decay heat in ${ }^{239} \mathrm{Pu}$ : Solving the $\gamma$ discrepancy in the 4-3000 s cooling period, Phys. Rev. Lett. 105 (2010) 202501. doi:10.1103/PhysRevLett.105.202501.

URL http://link.aps.org/doi/10.1103/PhysRevLett.105.202501

[9] A. Simon, S. Quinn, A. Spyrou, A. Battaglia, I. Beskin, A. Best, B. Bucher, M. Couder, P. DeYoung, X. Fang, J. Görres, A. Kontos, Q. Li, S. Liddick, A. Long, S. Lyons, K. Padmanabhan, J. Peace, A. Roberts, D. Robertson, K. Smith, M. Smith, E. Stech, B. Stefanek, W. Tan, X. Tang, M. Wiescher, SuN: Summing $\mathrm{NaI}(\mathrm{Tl})$ gamma-ray detector for capture reaction measurements, Nuclear Instruments and Methods A 703 (0) (2013) 16 21.

[10] J. Tain, A. Algora, J. Agramunt, V. Guadilla, M. Jordan, A. MontanerPizá, B. Rubio, E. Valencia, D. Cano-Ott, W. Gelletly, T. Martinez, E. Mendoza, Z. Podolyák, P. Regan, J. Simpson, A. Smith, J. Strachan, A decay total absorption spectrometer for DESPEC at FAIR, Nuclear Instruments and Methods A 803 (2015) 36-46.

[11] NEA, Assessment of Fission Product Decay Data for Decay Heat Calculations: A report by the Working Party on International Evaluation Cooperation of the Nuclear Energy Agency Nuclear Science Committee, Nuclear Energy Agency, Organization for Economic Co-operation and Development, Paris, France, 2007. 
[12] X. LLC, User's Manual Digital Gamma Finder (DGF) Pixie-16, Version 1.40 (October 2009).

[13] D. Cano-Ott, J. L. Tain, A. Gadea, B. Rubio, L. Batist, M. Karny, E. Roeckl, Monte Carlo simulation of the response of a large $\mathrm{NaI}(\mathrm{Tl})$ total absorption spectrometer for beta-decay studies, Nuclear Instruments and Methods A 430 (1999) 333-347.

[14] S. Agostinelli, J. Allison, K. Amako, J. Apostolakis, H. Araujo, P. Arce, M. Asai, D. Axen, S. Banerjee, G. Barrand, F. Behner, L. Bellagamba, J. Boudreau, L. Broglia, A. Brunengo, H. Burkhardt, S. Chauvie, J. Chuma, R. Chytracek, G. Cooperman, G. Cosmo, P. Degtyarenko, A. Dell'Acqua, G. Depaola, D. Dietrich, R. Enami, A. Feliciello, C. Ferguson, H. Fesefeldt, G. Folger, F. Foppiano, A. Forti, S. Garelli, S. Giani, R. Giannitrapani, D. Gibin, J. G. Cadenas, I. González, G. G. Abril, G. Greeniaus, W. Greiner, V. Grichine, A. Grossheim, S. Guatelli, P. Gumplinger, R. Hamatsu, K. Hashimoto, H. Hasui, A. Heikkinen, A. Howard, V. Ivanchenko, A. Johnson, F. Jones, J. Kallenbach, N. Kanaya, M. Kawabata, Y. Kawabata, M. Kawaguti, S. Kelner, P. Kent, A. Kimura, T. Kodama, R. Kokoulin, M. Kossov, H. Kurashige, E. Lamanna, T. Lampén, V. Lara, V. Lefebure, F. Lei, M. Liendl, W. Lockman, F. Longo, S. Magni, M. Maire, E. Medernach, K. Minamimoto, P. M. de Freitas, Y. Morita, K. Murakami, M. Nagamatu, R. Nartallo, P. Nieminen, T. Nishimura, K. Ohtsubo, M. Okamura, S. O’Neale, Y. Oohata, K. Paech, J. Perl, A. Pfeiffer, M. Pia, F. Ranjard, A. Rybin, S. Sadilov, E. D. Salvo, G. Santin, T. Sasaki, N. Savvas, Y. Sawada, S. Scherer, S. Sei, V. Sirotenko, D. Smith, N. Starkov, H. Stoecker, J. Sulkimo, M. Takahata, S. Tanaka, E. Tcherniaev, E. S. Tehrani, M. Tropeano, P. Truscott, H. Uno, L. Urban, P. Urban, M. Verderi, A. Walkden, W. Wander, H. Weber, J. Wellisch, T. Wenaus, D. Williams, D. Wright, T. Yamada, H. Yoshida, D. Zschiesche, GEANT4 - a simulation toolkit, Nuclear Instruments and Methods A 506 (2003) 250-303.

[15] B. C. Rasco, A. Fijałkowska, M. Karny, K. P. Rykaczewski, M. WolińskaCichocka, R. Grzywacz, K. C. Goetz, The nonlinear output of $\mathrm{NaI}(\mathrm{Tl})$ detectors in Modular Total Absorption Spectrometer, Nuclear Instruments and Methods A 788 (2015) 137-145.

[16] A. Gilbert, A. G. W. Cameron, A composite nulcear-level density formula with shell corrections, Canadian Journal of Physics 43 (8) (1965) 14461496.

[17] J. L. Tain, D. Cano-Ott, The influence of the unknown de-excitation pattern in the analysis of -decay total absorption spectra, Nuclear Instruments and Methods A 571 (2007) 719-727.

[18] J. L. Tain, D. Cano-Ott, Algorithms for the analysis of $\beta$-decay total absorption spectra, Nuclear Instruments and Methods A 571 (2007) 728-738. 
[19] B. C. Rasco, A. Fijałkowska, M. Karny, K. P. Rykaczewski, M. WolińskaCichocka, K. C. Goetz, R. Grzywacz, C. Gross, K. Miernik, S. Pulauskas, Multiple $\gamma$ emission of the ${ }^{137}$ Xe 2849-2850keV levels studied with Modular Total Absorption Spectrometer (MTAS), JPS Conference Proceedings 6 (030018).

[20] R. M. Yee, N. D. Scielzo, P. F. Bertone, F. Buchinger, S. Caldwell, J. A. Clark, C. M. Deibel, J. Fallis, J. P. Greene, S. Gulick, D. Lascar, A. F. Levand, G. Li, E. B. Norman, M. Pedretti, G. Savard, R. E. Segel, K. S. Sharma, M. G. Sternberg, J. Van Schelt, B. J. Zabransky, $\beta$-delayed neutron spectroscopy using trapped radioactive ions, Phys. Rev. Lett. 110 (2013) 092501.

[21] H. Ohm, M. Zendel, S. Prussin, W. Rudolph, A. Schroder, K.-L. Kratz, C. Ristori, J. Pinston, E. Monnand, F. Schussler, J. Zirnheld, Beta-Delayed Neutrons and High-Energy Gamma-Rays from Decay of ${ }^{137}$ I, Z.Phys A296 (1980) 23. 